\title{
Quantum Hall effects in a non-Abelian honeycomb lattice
}

\author{
Ling Li, ${ }^{1}$ Ningning Hao, ${ }^{2}$ Guocai Liu,,${ }^{1}{ }^{*}$ Zhiming Bai, ${ }^{1}$ Zai-Dong Li, ${ }^{3}$ Shu Chen, ${ }^{4}$ and W. M. Liu ${ }^{4}$ \\ ${ }^{1}$ School of Science, Hebei University of Science and Technology, Shijiazhuang 050018, China \\ ${ }^{2}$ Department of Physics, The University of Hong Kong, Pokfulam Road, Hong Kong, China \\ ${ }^{3}$ Department of Applied Physics, Hebei University of Technology, Tianjin 300401, China \\ ${ }^{4}$ Beijing National Laboratory for Condensed Matter Physics, Institute of Physics, Chinese Academy of Science, Beijing 100190, China
}

(Received 15 May 2015; revised manuscript received 30 September 2015; published 10 December 2015)

\begin{abstract}
We study the tunable quantum Hall effects in a non-Abelian honeycomb optical lattice which is a multiDirac-point system. We find that the quantum Hall effects present different features with the change in relative strengths of several perturbations. Namely, the quantum spin Hall effect can be induced by gauge-field-dressed next-nearest-neighbor hopping, which, together with a Zeeman field, can induce the quantum anomalous Hall effect characterized by different Chern numbers. Furthermore, we find that the edge states of the multi-Diracpoint system represent very different features for different boundary geometries, in contrast with the generic two-Dirac-point system. Our study extends the borders of the field of quantum Hall effects in a honeycomb optical lattice with multivalley degrees of freedom.
\end{abstract}

DOI: 10.1103/PhysRevA.92.063618

PACS number(s): 03.75.Lm, 73.43.Nq

\section{INTRODUCTION}

The honeycomb lattice, which is the brick that builds graphene, plays a significant role in promoting new physics. The most remarkable feature of the energy band of the honeycomb lattice system is that the low-energy excitations display a linear dispersion relation [1] and are, thus, described by massless Dirac fermions [2]. Furthermore, massless Dirac fermions can be tuned into massive Dirac fermions by tunable perturbations. More importantly, the transition from the gapless to the gapped phase can result in new physics, such as the quantum anomalous Hall (QAH) effect in the well-known Haldane graphene model [3] and quantum spin Hall (QSH) effect in the Kane-Mele graphene model [4]. The former model pioneers a new route to realize non-zero-integer Hall conductance without Landau levels and the latter model establishes the foundation to breed topological insulators $[5,6]$. Comparison between the two models indicates the power of the internal spin degrees of freedom to produce new physics in honeycomb lattice systems.

On the other hand, the development of laser and ultracoldatom techniques gives rise to various optical lattices in which ultracold Fermi atoms can be trapped to simulate the phenomena in condensed-matter systems [7-13], such as the topological Mott insulator (MI) [14] and superfluid (SF)-MI transition $[15,16]$. More significantly, in a laser field with a specific configuration, the trapped ultracold atoms can feel effective Abelian or non-Abelian gauge fields [17,18]. The couplings between the trapped ultracold atoms and the artificial gauge fields are equivalent to various interactions in cold-atom systems, such as spin-orbital couplings $[19,20]$.

In this paper, we study a non-Abelian honeycomb optical lattice which is a two-dimensional multi-Dirac-point system. Compared with the usual honeycomb lattice, in which only two independent Dirac points emerge, the non-Abelian honeycomb optical lattice here has eight Dirac points. Namely, the dimension of the internal valley degrees of freedom is extended

\footnotetext{
*guocailiu@semi.ac.cn
}

from 2 to 8 , and the extended valley degrees of freedom are induced by the couplings between the trapped atoms and the non-Abelian gauge fields. Likewise, we introduce three kinds of perturbations to open a gap of Dirac points independently. Explicitly, two perturbations including staggered sublattice potentials and gauge-field-dressed next-nearest-neighbor (NNN) hopping are time reversal invariant. The third is Zeeman splitting, which breaks the time-reversal symmetry of the system. We find that staggered sublattice potentials give a trivial gapped phase with no gapless edge states in the ribbon geometry, and the gauge-field-dressed NNN hopping results in the QSH effect, with the spin direction lying in the ribbon plane. Interestingly, we find that the edge states connecting different internal valleys present different features even though the overall Chern number is 0 in the presence of both staggered sublattice potentials and Zeeman splitting. Furthermore, it is shown that cooperations among the three kinds of perturbations can induce QAH effects characterized by different Chern numbers. Likewise, edge states show internal structures for different types of edge boundaries. The results indicate that many-Dirac-point systems represent more abundant physics in comparison with simple two-Dirac-point systems, and the robustness of the bulk-boundary correspondence is global, not local.

The paper is organized as follows. In Sec. II, we introduce the non-Abelian honeycomb lattice model with three kinds of perturbations. In Sec. III, we study the topological phase transition driven by three kinds of perturbations by investigating the change in the valley Chern numbers defined at Dirac points. Furthermore, we study the relations between the change in Chern numbers and the emergence of edge states under different geometries. In Sec. IV, we discuss some important relevant issues and give a brief summary.

\section{MODEL}

We start with a non-Abelian honeycomb lattice model proposed in Ref. [21] and introduce three kinds of perturbations into this model to open a gap at each Dirac point, respectively. 
The tight-binding Hamiltonian takes the form

$$
H=H_{0}+H_{1},
$$

with

$$
\begin{gathered}
H_{0}=-t \sum_{\langle i, j\rangle} b_{j}^{\dagger} U_{i j} a_{i}+\text { H.c. } \\
H_{1}=-t_{1} \sum_{\langle\langle i, j\rangle\rangle}\left(a_{j}^{\dagger} U_{i j}^{\prime} a_{i}+b_{j}^{\dagger} U_{i j}^{\prime} b_{i}\right)+\delta \sum_{i}\left(a_{i}^{\dagger} a_{i}-b_{i}^{\dagger} b_{i}\right) \\
+h \sum_{i}\left(a_{i}^{\dagger} \sigma_{z} a_{i}+b_{i}^{\dagger} \sigma_{z} b_{i}\right)
\end{gathered}
$$

Here $t\left(t_{1}\right)$ is the hopping amplitude between the (next-) nearest-neighbor link $\langle i, j\rangle \quad(\langle\langle i, j\rangle\rangle), a_{i}^{\dagger}=\left(a_{i \uparrow}^{\dagger}, a_{i \downarrow}^{\dagger}\right)$, with $a_{i \alpha}^{\dagger}\left(a_{i \alpha}\right)$ denoting the creation (annihilation) operator of a fermionic atom with spin $\alpha$ (up or down) at A-sublattice $i$ (a similar definition applies for sublattice B). The unitary operator $U_{i j}\left(U_{i j}^{\prime}\right)$ is associated with the link connecting the (next-) nearest-neighbor lattice points $\mathbf{r}_{i} \rightarrow \mathbf{r}_{j}$ [21]. The unitary operators coupling fermionic atoms to non-Abelian fields along each hopping path from sublattice A to sublattice $\mathrm{B}$ are taken as $U_{1}=e^{i \alpha_{1} \sigma_{x}}, U_{2}=1, U_{3}=e^{i \alpha_{3} \sigma_{y}}$. The three nearest-neighbor hopping pathes are $\mathbf{d}_{1}=a\left(\frac{\sqrt{3}}{2},-\frac{1}{2}\right), \mathbf{d}_{2}=$ $a(0,1), \mathbf{d}_{3}=a\left(-\frac{\sqrt{3}}{2},-\frac{1}{2}\right)$. Similarly, the unitary operators along each hopping path between the NNN lattice points are chosen as $U_{1}^{\prime}=U_{4}^{\prime \dagger}=e^{i \gamma_{1} \sigma_{z}}, U_{2}^{\prime}=U_{5}^{\prime \dagger}=e^{i \gamma_{2} \sigma_{z}}, U_{3}^{\prime}=$ $U_{6}^{\prime \dagger}=e^{i \gamma_{3} \sigma_{z}}$, with NNN hopping paths $\mathbf{b}_{1}=-\mathbf{b}_{4}=a(\sqrt{3}, 0)$, $\mathbf{b}_{2}=-\mathbf{b}_{5}=a\left(\frac{\sqrt{3}}{2}, \frac{3}{2}\right), \mathbf{b}_{3}=-\mathbf{b}_{6}=a\left(-\frac{\sqrt{3}}{2}, \frac{3}{2}\right)$. Here, $\alpha_{s=1,3}$ or $\gamma_{s=1,2,3}$ is the gauge flux, and $\sigma_{x}, \sigma_{y}$, and $\sigma_{z}$ are the Pauli matrices for spin. $\delta$ denotes an on-site energy for sublattice A and $\mathrm{B}$, and $h$ is the Zeeman splitting. For simplicity, we choose $t=1$ as the energy unit and the distance $a$ between the nearest sites as the length unit throughout this paper.

In the momentum space, Hamiltonian (1) can be written in the basis $\psi_{\mathbf{k}}=\left(a_{\mathbf{k} \uparrow}, b_{\mathbf{k} \uparrow}, a_{\mathbf{k} \downarrow}, b_{\mathbf{k} \downarrow}\right)^{T}$ :

$$
H=\sum_{\mathbf{k}} \psi_{\mathbf{k}}^{\dagger}\left[\mathcal{H}_{0}(\mathbf{k})+\mathcal{H}_{1}(\mathbf{k})\right] \psi_{\mathbf{k}},
$$

with

$$
\mathcal{H}_{0}(\mathbf{k})=\left(\begin{array}{cccc}
0 & -t P_{0}^{*}(\mathbf{k}) & 0 & -t P_{2}^{*}(\mathbf{k}) \\
-t P_{0}(\mathbf{k}) & 0 & -t P_{1}(\mathbf{k}) & 0 \\
0 & -t P_{1}^{*}(\mathbf{k}) & 0 & -t P_{0}^{*}(\mathbf{k}) \\
-t P_{2}(\mathbf{k}) & 0 & -t P_{0}(\mathbf{k}) & 0
\end{array}\right)
$$

$$
\mathcal{H}_{1}(\mathbf{k})=-\left(t_{1} P_{3}(\mathbf{k})-h\right)\left(\sigma_{z} \otimes \tau_{0}\right)+\delta\left(\sigma_{0} \otimes \tau_{z}\right),
$$

and

$$
\begin{aligned}
& P_{0}(\mathbf{k})=e^{-i \mathbf{k} \cdot \mathbf{d}_{2}}, \\
& P_{1}(\mathbf{k})=i e^{-i \mathbf{k} \cdot \mathbf{d}_{1}}+e^{-i \mathbf{k} \cdot \mathbf{d}_{3}}, \\
& P_{2}(\mathbf{k})=i e^{-i \mathbf{k} \cdot \mathbf{d}_{1}}-e^{-i \mathbf{k} \cdot \mathbf{d}_{3}}, \\
& P_{3}(\mathbf{k})=2 \sum_{s=1,2,3} \sin \left(\mathbf{k} \cdot \mathbf{b}_{s}\right) .
\end{aligned}
$$

(a)
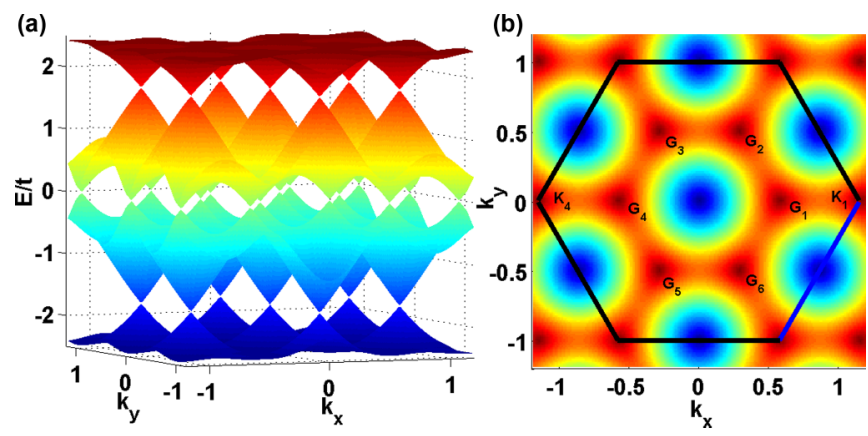

FIG. 1. (Color online) (a) Band structures for the Hamiltonian $H_{0}$ with $\alpha_{s=1,3}=\frac{\pi}{2}$. (b) Constant energy surfaces corresponding to the second band. The Dirac points for the middle two bands lie at the $\mathbf{K}_{s=1, \ldots, 6}$ points and $\mathbf{G}_{s=1, \ldots, 6}$ points in the first Brillouin zone. Here, we only mark $\mathbf{K}_{1}$ and $\mathbf{K}_{4}$ and choose $\frac{2 \pi}{3}$ as the unit of $k_{x}$ and $k_{y}$.

Here, we set $\alpha_{s=1,3}=\gamma_{s=1,2,3}=\frac{\pi}{2} . \tau_{z}$ is the Pauli matrix to span two sublattices. $\tau_{0}$ and $\sigma_{0}$ are $2 \times 2$ unit matrices. The energy spectrum for the Hamiltonian $\mathcal{H}_{0}(\mathbf{k})$ is shown in Fig. 1(a), where one sees that the whole energy spectrum displays a "particle-hole" symmetry with respect to the Fermi energy $\epsilon_{F}=0$ and any two adjacent touching bands exhibit Dirac-type energy spectra. The lower (upper) two bands touch at six $\mathbf{M}$ points in the first Brillouin zone. The middle two bands touch at six $\mathbf{K}$ points, only two of which are independent, with $\mathbf{K}_{1,4}=\frac{2 \pi}{3}\left( \pm \frac{2 \sqrt{3}}{3}, 0\right), \mathbf{K}_{2,3,5,6}=\frac{2 \pi}{3}\left( \pm \frac{\sqrt{3}}{3}, \pm 1\right)$, and $\mathbf{G}$ points with $\mathbf{G}_{1,4}=\frac{2 \pi}{3}\left( \pm \frac{\sqrt{3}}{3}, 0\right), \mathbf{G}_{2,3,5,6}=\frac{2 \pi}{3}\left( \pm \frac{\sqrt{3}}{6}, \pm \frac{1}{2}\right)$ in the first Brillouin zone [shown in Fig. 1(b)]. Here, we choose $\frac{2 \pi}{3}$ as the unit of $k_{x}$ and $k_{y}$ in all of the figures in this paper.

\section{QUANTUM HALL EFFECTS INDUCED BY PERTURBATIONS}

To open a gap at each Dirac point, $\mathcal{H}_{1}(\mathbf{k})$ in Eq. (4) is turned on. The scheme for simulation of the gauge field with $\gamma_{s=1,2,3}=\frac{\pi}{2}$ is presented in the Appendix. It is straightforward to check that nonzero $t_{1}, \delta$, and $h$ can independently open gaps at the eight inequivalent Dirac points. However, gaps from different perturbations may have different topological features, and the competitions or cooperations between these perturbations determine the overall topological properties of the system. In order to explicitly show the connections between the topological feature and the amplitudes of perturbations, we expand the Hamiltonian in Eqs. (5) and (6) around each Dirac point and obtain the relevant low-energy Hamiltonian as follows:

$$
\mathcal{H}^{(m)}(\mathbf{k})=d_{x}^{(m)}(\mathbf{k}) \eta_{x}+d_{y}^{(m)}(\mathbf{k}) \eta_{y}+d_{z}^{(m)}(\mathbf{k}) \eta_{z} .
$$

Here, $\eta_{x}, \eta_{y}$, and $\eta_{z}$ are the Pauli matrices in the effective band basis. $m$ labels the Dirac point, and $\mathbf{k}$ is measured from the Dirac points. We summarize three components $\left[d_{x}^{(m)}(\mathbf{k}), d_{y}^{(m)}(\mathbf{k}), d_{z}^{(m)}(\mathbf{k})\right]$ of $\hat{\mathbf{d}}^{(m)}(\mathbf{k})$ for all the Dirac points in Table I.

The valley Chern number of the lower band around Dirac point $m$ can be calculated by [22]

$$
C_{m}=\frac{1}{2} \operatorname{sgn}\left(\partial_{k_{x}} \hat{\mathbf{d}}^{(m)}(\mathbf{k}) \times \partial_{k_{y}} \hat{\mathbf{d}}^{(m)}(\mathbf{k})\right)_{z} \operatorname{sgn}\left(d_{z}^{(m)}\right),
$$


TABLE I. Coefficients of the Dirac Hamiltonian at eight Dirac points.

\begin{tabular}{lccc}
\hline \hline & $d_{x}(\mathbf{k})$ & $d_{y}(\mathbf{k})$ & $d_{z}(\mathbf{k})$ \\
\hline $\mathbf{K}_{1} / \mathbf{G}_{4}$ & $-\frac{3 t k_{x}}{\sqrt{6}}$ & $\frac{3 t k_{y}}{\sqrt{6}}$ & $\left(\delta-t_{1}-\frac{\sqrt{3} h}{3}\right)$ \\
$\mathbf{K}_{4} / \mathbf{G}_{1}$ & $\frac{3 t k_{x}}{\sqrt{6}}$ & $\frac{3 t k_{y}}{\sqrt{6}}$ & $\left(\delta-t_{1}+\frac{\sqrt{3} h)}{3}\right)$ \\
$\mathbf{G}_{2} / \mathbf{G}_{5}$ & $\mp \frac{\sqrt{6} t\left(k_{x}-\sqrt{3} k_{y}\right)}{4}$ & $\frac{\sqrt{6} t\left(\sqrt{3} k_{x}+k_{y}\right)}{4}$ & $\left(\delta-3 t_{1} \pm \frac{\sqrt{3} h}{3}\right)$ \\
$\mathbf{G}_{3} / \mathbf{G}_{6}$ & $\pm \frac{\sqrt{6} t\left(k_{x}+\sqrt{3} k_{y}\right)}{4}$ & $\frac{\sqrt{6} t\left(-\sqrt{3} k_{x}+k_{y}\right)}{4}$ & $\left(\delta+t_{1} \mp \frac{\sqrt{3} h}{3}\right)$ \\
\hline \hline
\end{tabular}

from which we obtain

$$
\begin{gathered}
C_{\mathbf{K}_{1} / \mathbf{G}_{4}}=-\frac{1}{2} \operatorname{sgn}\left(\delta-t_{1}-\frac{\sqrt{3}}{3} h\right), \\
C_{\mathbf{K}_{4} / \mathbf{G}_{1}}=\frac{1}{2} \operatorname{sgn}\left(\delta-t_{1}+\frac{\sqrt{3}}{3} h\right), \\
C_{\mathbf{G}_{2} / \mathbf{G}_{5}}=\mp \frac{1}{2} \operatorname{sgn}\left(\delta-3 t_{1} \pm \frac{\sqrt{3}}{3} h\right), \\
C_{\mathbf{G}_{3} / \mathbf{G}_{6}}= \pm \frac{1}{2} \operatorname{sgn}\left(\delta+t_{1} \mp \frac{\sqrt{3}}{3} h\right) .
\end{gathered}
$$

Having obtained the expressions for the valley Chern numbers, we now discuss the influences of the perturbations on the topological properties of the system. Generally speaking, the topological properties are the global features of the system, and some physical quantities have simple corresponding relations to the total Chern numbers, such as the Hall conductance $\sigma_{x y}^{H}=-\frac{e^{2}}{h} \sum_{m} C_{m}$ in the electron system and the mass conductance $\sigma_{x y}^{M} \propto \sum_{m} C_{m}$ in the ultracold-atom system, with $\delta C_{m}$ denoting the change in Chern number with the applied perturbations. For the two-Dirac-point system, $C_{m}$ can only take the value 0 or \pm 1 , and the bulk-boundary correspondence is simple. For the multi-Dirac-point system here, $C_{m}$ can take a series of values, and the bulk-edge correspondence can represent abundant features due to the extended internal valley degrees of freedom. Furthermore, edge states can show different features for different boundaries.

In order to present an explicit picture of the bulk-boundary correspondence in the multi-Dirac-point system, we calculate the spectra of Hamiltonian (1) with zigzag (armchair) boundaries along the $y(x)$ direction and periodic boundaries along the $x(y)$ direction. The results are shown in Fig. 2 and 3 . For a ribbon with zigzag boundaries, by comparing the magnitudes of the gaps opened by $t_{1}, \delta$, and $h$ at each Dirac point, we can identify that the points $\left(\mathbf{G}_{\mathbf{3}}, \mathbf{G}_{\mathbf{5}}\right)$ in the bulk Brillouin zone are projected to the same momentum $k_{x}=-\frac{\sqrt{3} \pi}{9}$ in Fig. 2. Similarly, the points $\left(\mathbf{G}_{\mathbf{2}}, \mathbf{G}_{\mathbf{6}}\right),\left(\mathbf{G}_{\mathbf{1}}, \mathbf{K}_{\mathbf{4}}\right)$, and $\left(\mathbf{K}_{\mathbf{1}}, \mathbf{G}_{\mathbf{4}}\right)$ are projected to the momenta $k_{x}=\frac{\sqrt{3} \pi}{9}, \frac{2 \sqrt{3} \pi}{9}$, and $\frac{4 \sqrt{3} \pi}{9}$, respectively. The same conclusions can also be

(b)

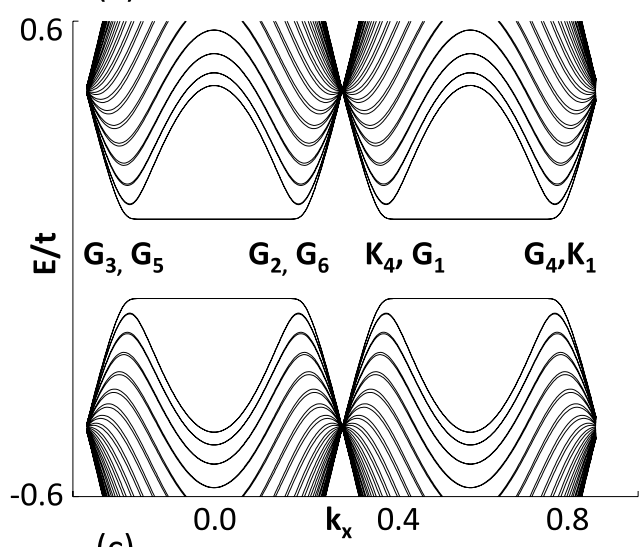

(c)

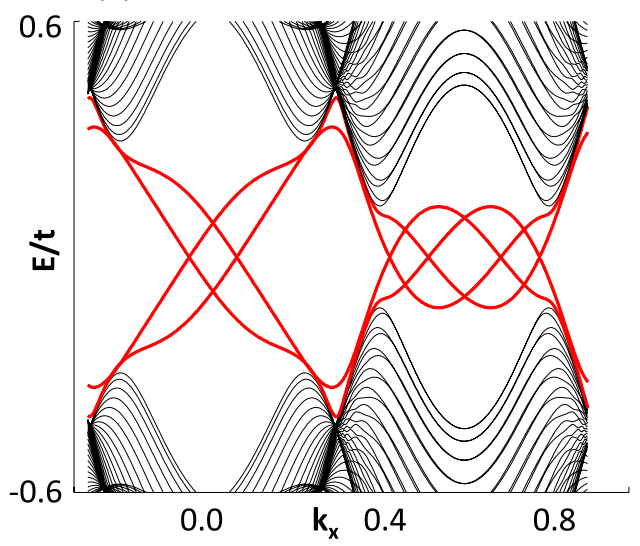

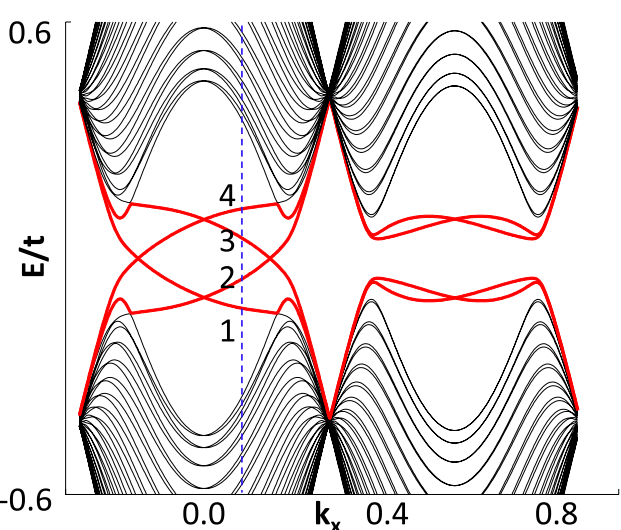

(d)

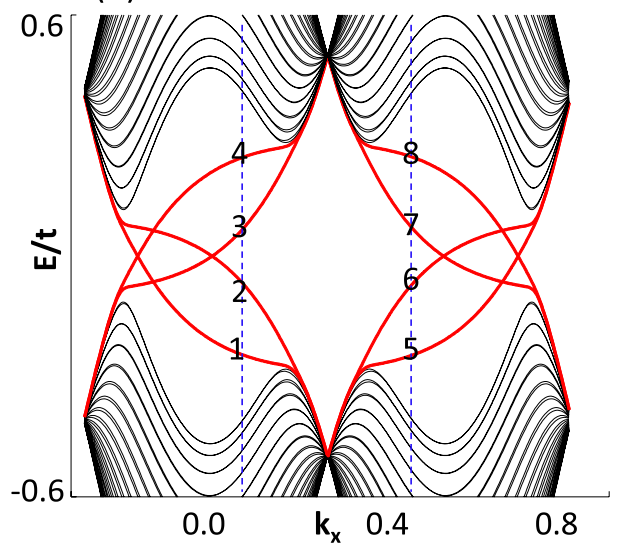

FIG. 2. (Color online) Band structures of Hamiltonian (1) in the ribbon geometry with zigzag edges and 40 sites in the $y$ direction, corresponding to (a) $\left(\delta, t_{1}, h\right)=(0.1,0,0)$, (b) $(0.1,0.05,0)$, (c) $(0.1,0.2,0)$, and (d) $(0.1,0,0.3)$. Here, $k_{x}$ is measured in units of $2 \pi / 3$. 
(a)
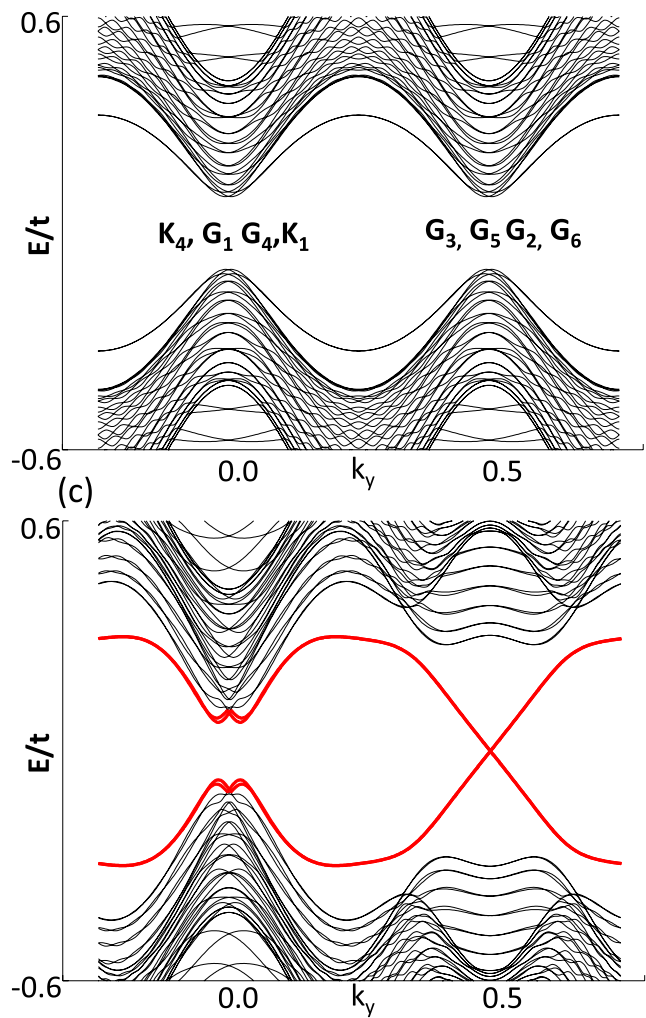

(b)
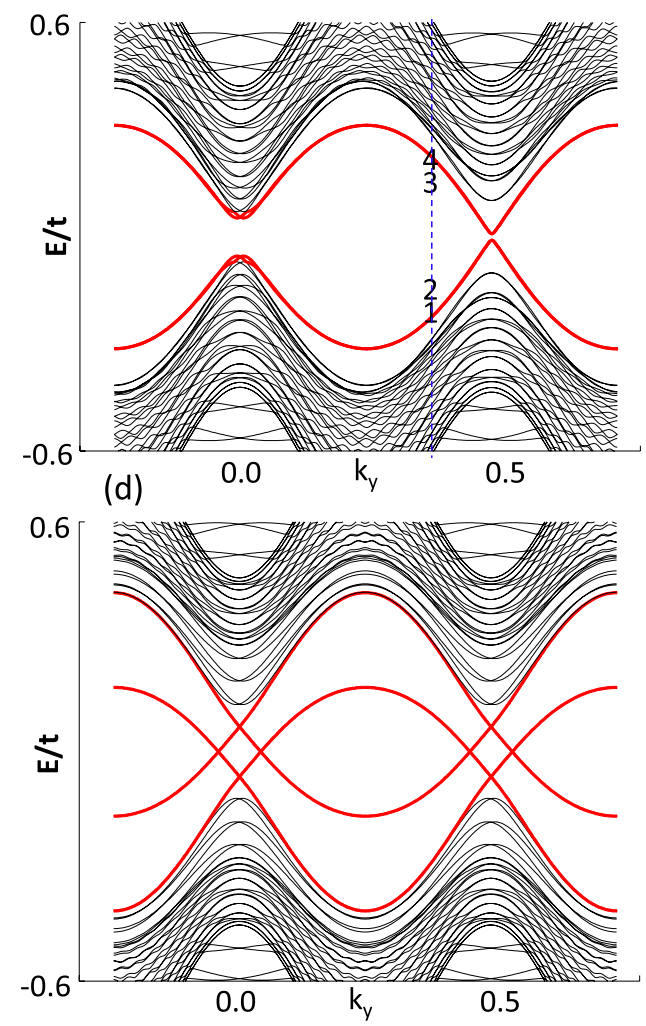

FIG. 3. (Color online) Band structures of Hamiltonian (1) in the ribbon geometry with armchair edges and 60 sites in the $x$ direction, corresponding to (a) $\left(\delta, t_{1}, h\right)=(0.1,0,0)$, (b) $(0.1,0.05,0)$, (c) $(0.1,0.2,0)$, and (d) $(0.1,0,0.3)$. Here, $k_{y}$ is measured in units of $2 \pi / 3$.

drawn from Fig. 1(b). Thus, it is convenient to divide the eight Dirac points into two groups, with each group involving four points. Explicitly, group I includes points $\left\{\mathbf{G}_{\mathbf{3}}, \mathbf{G}_{\mathbf{2}}\right.$, $\left.\mathbf{G}_{\mathbf{5}}, \mathbf{G}_{\mathbf{6}}\right\}$, and group II includes points $\left\{\mathbf{G}_{\mathbf{1}}, \mathbf{K}_{\mathbf{1}}, \mathbf{K}_{\mathbf{4}}, \mathbf{G}_{\mathbf{4}}\right\}$. See Fig. 2(a) for details. Furthermore, each group can be divided into two subgroups and each subgroup includes two Dirac points. Namely, two subgroups in group I are $\left\{\mathbf{G}_{\mathbf{3}}, \mathbf{G}_{\mathbf{2}}\right\}$ and $\left\{\mathbf{G}_{\mathbf{5}}, \mathbf{G}_{\mathbf{6}}\right\}$, while two subgroups in group II are $\left\{\mathbf{G}_{\mathbf{1}}, \mathbf{K}_{\mathbf{1}}\right\}$ and $\left\{\mathbf{K}_{\mathbf{4}}, \mathbf{G}_{\mathbf{4}}\right\}$. The topological properties of Dirac points can be characterized by the valley Chern numbers $C_{\mathbf{G}_{\mathrm{i}}}$ and $C_{\mathbf{K}_{\mathbf{i}}}$. In order to guarantee the bulk-boundary correspondence, we define the joint valley Chern numbers $C_{32}, C_{56}, C_{11}$, and $C_{44}$, in which $C_{32}=C_{\mathbf{G}_{3}}+C_{\mathbf{G}_{2}}$. Likewise, $C_{56}, C_{11}$, and $C_{44}$ have similar definitions. For a ribbon with armchair boundaries, with a similar strategy, we find that groups I and II are the same as in the case of a ribbon with zigzag boundaries. However, we find from Fig. 3(a) that the four Dirac points in each group mix together. We show that double-pairing and quadruple-pairing of Dirac points under different boundary conditions strongly influence the transport behaviors along different types of edges. The information on the edge states can be extracted from the distribution of the number and spin density of the atoms along the edges. Define the number and spin density of the atoms as follows:

$$
\begin{gathered}
n_{\alpha}^{(n)}(i)=\left\langle g\left|\alpha_{n}^{\dagger}(i) \sigma_{0} \alpha_{n}(i)\right| g\right\rangle, \\
s_{\alpha, \tau}^{(n)}(i)=\frac{1}{2}\left\langle g\left|\alpha_{n}^{\dagger}(i) \sigma_{\tau} \alpha_{n}(i)\right| g\right\rangle .
\end{gathered}
$$

Here, $n_{\alpha}^{(n)}(i)$ and $s_{\alpha, \tau}^{(n)}(i)$ denote the number and spin of atoms at site $i$ in the $n$th edge state. $\alpha=a, b$ marks two sublattices and $\tau=x, y, z$ labels three components of spin. $\alpha_{n}(i)=\left[\alpha_{n, \uparrow}(i), \alpha_{n, \downarrow}(i)\right]^{T}$.

Now, we discuss the evolution of the bulk-boundary correspondence by turning on different perturbations. For a ribbon with zigzag boundaries, starting with the nonzero $\delta$, the edge spectrum is shown in Fig. 2(a); no gapless edge states emerge. Correspondingly, the joint valley Chern number $C_{32}=C_{56}=C_{11}=C_{44}=0$. The system lies in a trivial state with the total Chern number $C_{\text {tot }}=C_{32}+C_{56}+C_{11}+C_{44}$ in the half-filling case. When $t_{1}$ is turned on, one can observe from Eqs. (9)-(12) that only $C_{\mathbf{G}_{2}}$ and $C_{\mathbf{G}_{5}}$ change signs when $t_{1} \in[\delta / 3, \delta]$. Then we have $C_{32}=\left.C_{\mathbf{G}_{2}}\right|_{t_{1}>\delta / 3}-\left.C_{\mathbf{G}_{2}}\right|_{t_{1}<\delta / 3}=$ +1 and $C_{56}=\left.C_{\mathbf{G}_{5}}\right|_{t_{1}>\delta / 3}-\left.C_{\mathbf{G}_{5}}\right|_{t_{1}<\delta / 3}=-1$. Meanwhile, two pairs of gapless edge states emerge [Fig. 2(b)], and each pair of edge states connects each pair of Dirac points $\left\{\mathbf{G}_{\mathbf{3}}, \mathbf{G}_{\mathbf{2}}\right\}$ and $\left\{\mathbf{G}_{\mathbf{5}}, \mathbf{G}_{\mathbf{6}}\right\}$, respectively. To show the features of edge states, we plot the distributions of $n_{\alpha}^{(n)}(i)$ and $s_{\alpha, \tau}^{(n)}(i)$ in Figs. 4(a1)-4(a4), and we can determine the propagation direction of the $n$th edge state through the velocity $v=\partial E_{n}\left(k_{x}\right) / \partial k_{x}$. The opposite propagation directions correspond to the opposite signs of the valley Chern numbers $C_{32}$ and $C_{56}$. The consistency between the valley Chern number $C_{32}, C_{56}$ and the gapless edge states satisfies the bulk-boundary correspondence. The transport pictures of the edge states are schematically illustrated in Fig. 4(c), where the edge states for $C_{32}=+1$ move along the clockwise direction and the edge states for $C_{56}=-1$ 

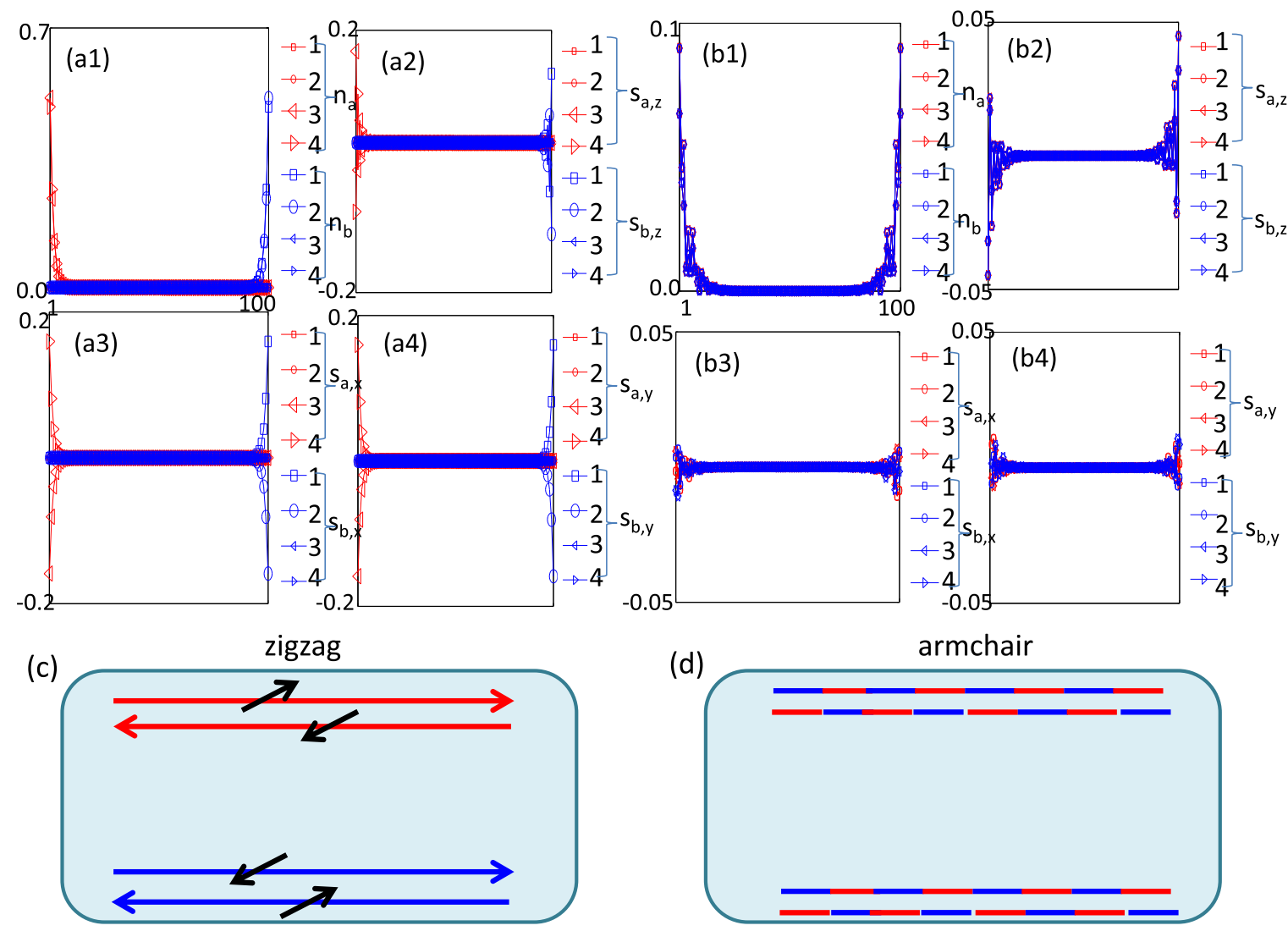

FIG. 4. (Color online) (a1)-(a4) [(b1)-(b4)] Distributions of the atom density $n_{\alpha}$ and three spin components $\left(s_{\alpha x}, s_{\alpha y}, s_{\alpha z}\right)$ along a zigzag [armchair] ribbon with 100 lattice sites for four edge states in Fig. 2(b) [Fig. 3(b)], labeled 1, 2, 3, and 4. Here, $\alpha=a, b$ denote two A and B sublattices. (c) Schematic of the movement of atoms in edge states shown in Fig. 2(b). The spin- $s_{z}$ component is nearly suppressed, while the spin- $s_{x}$ and $-s_{y}$ components have high weights. (d) Schematic of the accumulations of atoms in edge states corresponding to Fig. 3(b). The spin polarization is strongly suppressed, and no net current can be driven.

move along the counterclockwise direction. Furthermore, from Figs. 4(a2)-4(a4), we find that $s_{a, z}^{(1)}(i)$ and $s_{a, z}^{(2)}(i)$ exponentially decay as the distance to the boundary with a positive-negative oscillation, while $s_{a, x / y}^{(1)}(i)$ and $s_{a, x / y}^{(2)}(i)$ exponentially and monotonously decay as the distance to the boundary. The $s_{b, \tau}^{(n)}(i)$ have similar behaviors. Thus, we clarify that the spin polarizations of edge states almost lie in the $a-b$ plane, edge states with opposite spin polarizations propagate at opposite velocities at each boundary of ribbon [see Fig. 4(c)], and states with $t_{1} \in[\delta / 3, \delta]$ are QSH states. Compared with the standard QSH state proposed in the Kane-Mele model [4], which involves four bands, the number of effective bands in our model is two, but the increased internal valley degrees of freedom compensate the minimum four-band requirement to induce QSH states. In other multi-Dirac-point models [23,24], similar QSH states might exist if the gaps can been opened by some special perturbations. Of more practical significance, Ref. [25] has studied the transport properties of a silicene model with two kinds of Rashba spin-orbit couplings [26], where the QSH phase has similar features to our model. When $t_{1}$ is tuned to be larger than $\delta$, we find that $C_{\mathbf{K}_{1}}, C_{\mathbf{G}_{4}}, C_{\mathbf{K}_{2}}$, and $C_{\mathbf{G}_{1}}$ in group II change their signs, and the joint valley Chern number $C_{11}=C_{44}=0$. Even though two pairs of edge states emerge [see Fig. 2(c)], they can be adiabatically tuned into the bulk. The topological properties are similar to the case with $t_{1} \in[\delta / 3, \delta]$. For a ribbon with armchair boundaries, the joint valley Chern numbers $C_{32}$ and $C_{56}$ merge into a single one, $C_{3}+C_{5}+C_{2}+C_{6}$, as shown in Fig. 3(a). They become indistinguishable, and the joint valley Chern numbers cannot be well defined. Correspondingly, the edge states also mix together in Figs. 4(b1)-4(b4). These can be called quasiedge states, because they cannot induce the net spin current but only accumulate atoms at the armchair edges. We schematically show the features of quasiedge states in Fig. 4(d). Actually, the different behaviors of the edge states between zigzag and armchair boundaries can be understood as follows. Zigzag boundaries have an A-sublattice terminal at one boundary and a B-sublattice terminal at the other boundary, while armchair boundaries have both A- and B-sublattice terminals at both boundaries. Figures 4(a1)-4(a4) show that the chirality of the edge state can only be well defined when the edge state is localized on one kind of sublattice. This is the physical reason for the difference between ribbons with zigzag and ribbons with armchair boundaries. These features indicate that the transport behaviors of edge states from different types of edges can be very different in multi-Dirac-point systems even though the global structures are characterized by a single topological number.

Now, we turn to the effect of Zeeman splitting. Suppose that the system lies in a trivial state initially with $\delta \neq 0$ 


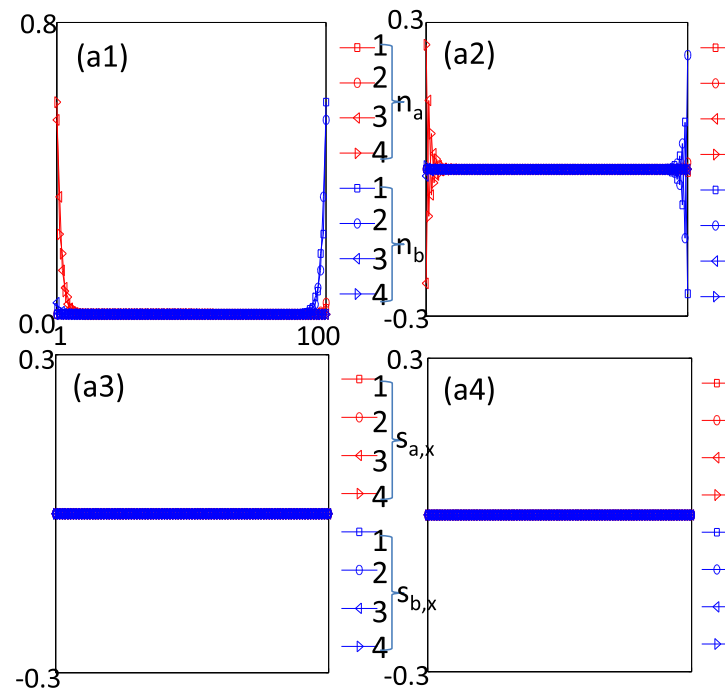

(c)

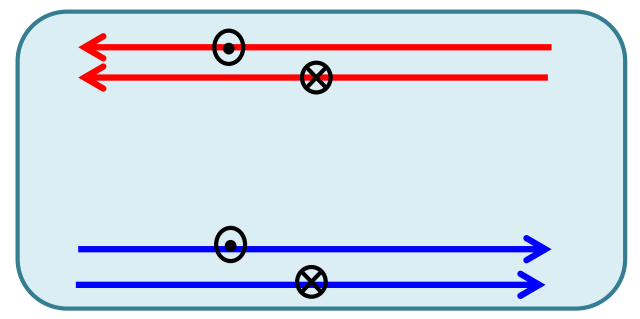

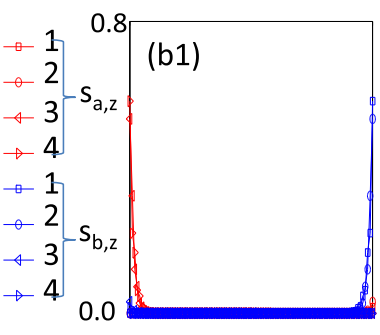
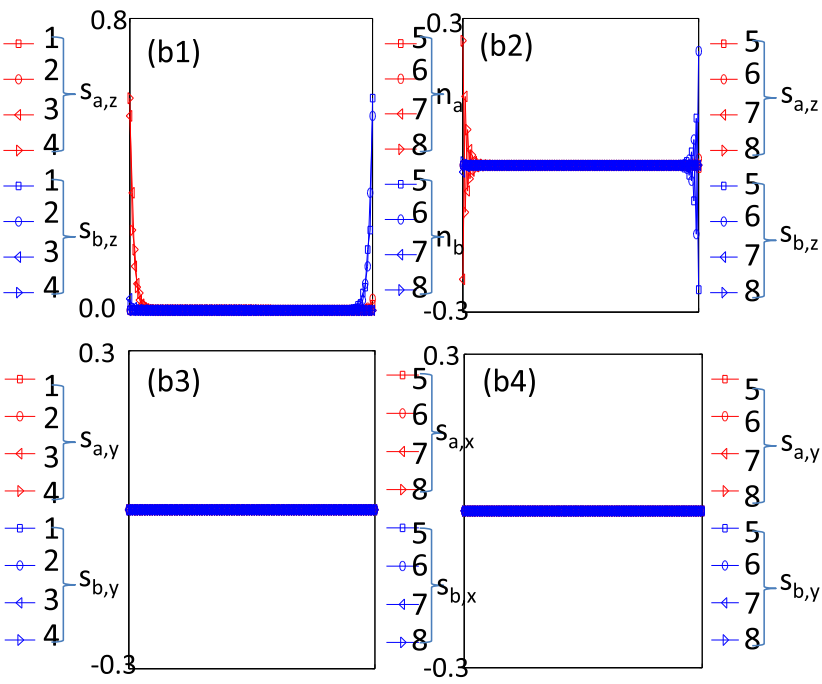

(d)

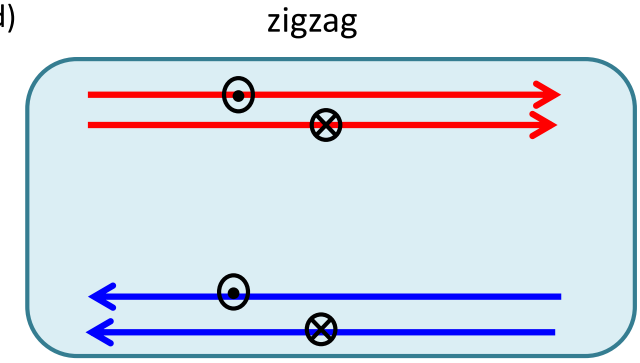

FIG. 5. (Color online) (a1)-(b4) Distributions of the atom density $n_{\alpha}$ and three spin components $\left(s_{\alpha x}, s_{\alpha y}, s_{\alpha z}\right)$ along a zigzag ribbon with 100 lattice sites for eight edge states in Fig. 2(d), labeled 1 to 8, respectively. Here, $\alpha=a, b$ denote two A and B sublattices. (c) Schematic of the movement of atoms in edge states in Fig. 2(d) labeled 1 to 4. (d) Schematic of the movement of atoms in edge states in Fig. 2(d) labeled 5 to 8 .

[Fig. 2(a)]. Turning to $h$, when $h>\sqrt{3} \delta, C_{\mathbf{K}_{1}}, C_{\mathbf{G}_{3}}, C_{\mathbf{G}_{4}}$, and $C_{\mathbf{G}_{5}}$ change their signs. For a ribbon with zigzag boundaries, straightforward calculations show that the joint valley Chern numbers $C_{32 / 56}=\left.C_{\mathbf{G}_{3 / 5}}\right|_{h>\sqrt{3} \delta}-\left.C_{\mathbf{G}_{3 / 5}}\right|_{h<\sqrt{3} \delta}=-1$ and $C_{44 / 11}=\left.C_{\mathbf{G}_{4} / \mathbf{K}_{1}}\right|_{h>\sqrt{3} \delta}-\left.C_{\mathbf{G}_{4} / \mathbf{K}_{1}}\right|_{h<\sqrt{3} \delta}=+1$, and there are four pairs of gapless edge states, shown in Fig. 2(d). Further analysis identifies that the two pairs of edge states connecting Dirac points in group I with $C_{32}=C_{56}=-1$ propagate along the counterclockwise direction and the edge states connecting Dirac points in group II with $C_{44}=C_{11}=+1$ propagate along the clockwise direction, as shown in Figs. 5(c)-5(d). In Figs. 5(a1)-5(b4), we see that $s_{a, x}^{(n)}(i)$ and $s_{a, y}^{(n)}(i)$ are 0 , while $s_{a, z}^{(n)}(i)$ is nonzero for $n=1 \ldots 8$, and the relevant spin polarization directions of the edge state are also shown in Figs. 5(c) and 5(d). Since the total Chern number is equal to 0 , one cannot distinguish this topological phase by measuring the Hall conductance. Figure 2(d) shows that the edge states are separated into two groups according to valleys with respect to $k_{x}=\frac{\sqrt{3} \pi}{6}$. Thus we call these quantum anomalous valley Hall states. If other perturbations or intervalley scatterings are introduced to break the topological structures in group I or II, the quantum anomalous valley Hall states become measurable. For a ribbon with armchair boundaries, the joint valley Chern number defined in the zigzag case shows the same behaviors, i.e., $C_{32}=C_{56}=-1$ and $C_{44}=C_{11}=+1$. Therefore, the quadruple-pairing of them does not induce destructive mixtures and the spectra of the edge states are shown in Fig. 3(d); they show features similar to those in Fig. 2(d), due to having the same joint valley Chern numbers $C_{32}=C_{56}=-1$ and $C_{44}=C_{11}=+1$.

Having obtained the individual pictures of nonzero $t_{1}$ and $h$, we turn to nonzero $t_{1}$ and nonzero $h$ combined. Then the Hall conductance or mass conductance becomes parameter dependent and is determined by the total Chern number. We plot the phase diagram about $t_{1} / t$ and $h / t$ with $\delta=0.1$ in Fig. 6, which shows that the Chern number can be changed from 0 to 2 by $t_{1}$ and $h$ in this multi-Dirac-point system. To clearly see the correspondence between the Chern number and the edge states of zigzag and armchair boundaries, we start with the parameters $\left(\delta, t_{1}, h\right)=(0.1,0.0,0.0)$ initially, and we have $C_{32}=C_{56}=C_{11}=C_{44}=0$. When $\left(\delta, t_{1}, h\right)=(0.1,0.03,0.11)$, only $C_{\mathbf{G}_{5}}$ changes its sign and we have $C_{56}=\left.C_{\mathbf{G}_{5}}\right|_{\delta<3 t_{1}+\frac{\sqrt{3}}{3} h}-\left.C_{\mathbf{G}_{5}}\right|_{\delta>3 t_{1}+\frac{\sqrt{3}}{3} h}=-1$. Thus, the system is in the QAH state with $C_{\text {tot }}=C_{56}=-1$. A pair of edge states emerges [see Figs. 7(a1) and 7(b1) for the cases of zigzag boundaries and armchair boundaries]. When $\left(\delta, t_{1}, h\right)=(0.1,0.03,0.18)$, then $C_{\mathbf{K}_{1}}$ and $C_{\mathbf{G}_{4}}$ also change signs. We obtain QAH states with $C_{44}=C_{11}=+1$ and the Chern number of the whole system $C=C_{56}+C_{44}+C_{11}=$ $-1+1+1=+1$. The corresponding three edge states are shown in Figs. 7(a2) and 7(b2); two pairs have positive chirality and one pair has negative chirality. Furthermore, when 


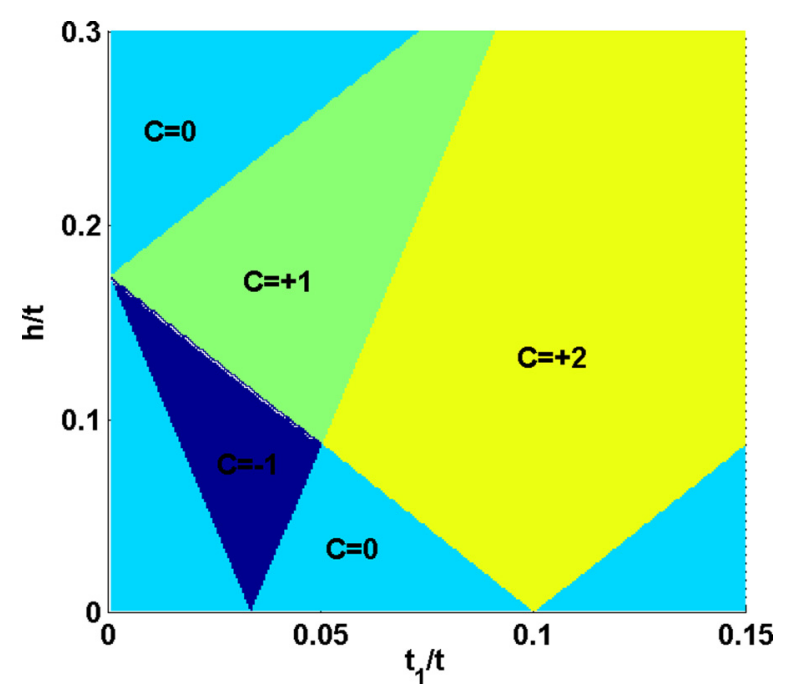

FIG. 6. (Color online) Phase diagram in the $t_{1}-h$ plane. Here, we set $\delta=0.1 t$.

$\left(\delta, t_{1}, h\right)=(0.1,0.1,0.07), C_{\mathbf{G}_{2}}$ changes sign and we obtain QAH states with $C=+2$. In Fig. 7(a3), we see that the edge states corresponding to $C_{56}=-1$ and $C_{23}=1$ couple with each other and open a gap to destroy nontrivial features, while the edge states corresponding to $C_{11}=1$ and $C_{44}=1$ show nontrivial features and retain the overall Chern number $C=+2$. Note that even the leftward two valleys in Fig. 3(b) have Chern numbers $C_{56}=-1$ and $C_{23}=1$, but no gap is opened between them in comparison with the edge states in
Fig. 7(a3). The reason is that the time-reversal symmetry is conserved in Fig. 3(b), and this symmetry protects the QSH state. On the other hand, the time-reversal symmetry is broken in Fig. 7(a3), and the two edge states can couple with each other and a band gap is opened. In Fig. 7(b3), it is straightforward to check that the two pairs of edge states at the quadruple-pairing Dirac points $\left\{\mathbf{G}_{\mathbf{3}}, \mathbf{G}_{\mathbf{2}}, \mathbf{G}_{\mathbf{5}}, \mathbf{G}_{\mathbf{6}}\right\}$ give the Chern number $C=$ $C_{3}+C_{5}+C_{2}+C_{6}=0$, and the overall Chern number $C=$ +2 is protected by another quadruple-pairing of Dirac points, $\left\{\mathbf{G}_{\mathbf{1}}, \mathbf{K}_{\mathbf{1}}, \mathbf{K}_{\mathbf{4}}, \mathbf{G}_{\mathbf{4}}\right\}$, with Chern number, $C=1+1=+2$. Note that in Fig. 7 (b3), the gapless edge states in the right valley are fake and not stable, and increasing Zeeman splitting can break these fake gapless edge states. Thus, the bulk-boundary correspondence is globally robust, however, the edge states represent different features for different boundaries.

\section{DISCUSSION AND SUMMARY}

In principle, the largest Chern number for this eight-Diracpoint system is 4 [22]. However, the phase diagram in Fig. 6 shows that the largest Chern number is 2 . For the present three kinds of perturbations, we cannot obtain a $C=4$ phase. If we introduce more perturbations to increase the numbers of parameters in Eqs. (8)-(11), we believe that the $C=4$ phase can be achieved. For example, the Chern numbers of $K_{1}, G_{4}, G_{2}$, and $G_{6}$ change signs, while the other four do not change signs. Thus, we can obtain the $C=4$ phase. We focus on three kinds of perturbations in the present paper and leave realizations of the higher Chern number phases for further studies.

In the electron system, the Hall conductance is well defined as the response coefficient of the electron current about (a1)

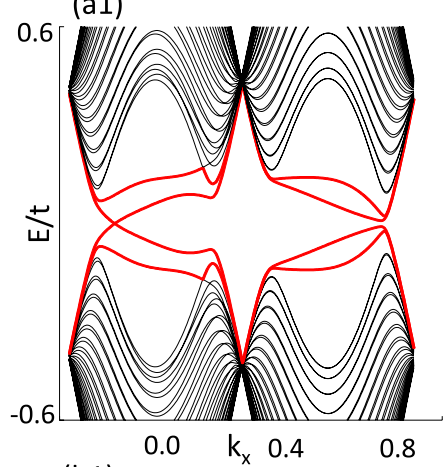

(b1)

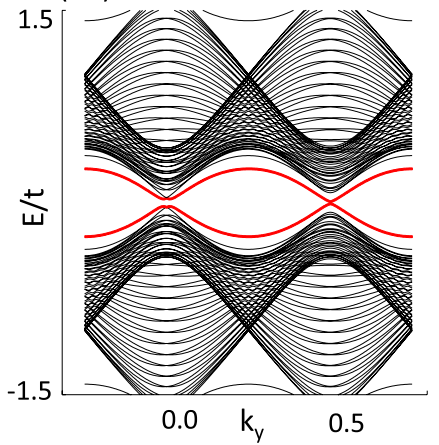

(a2)

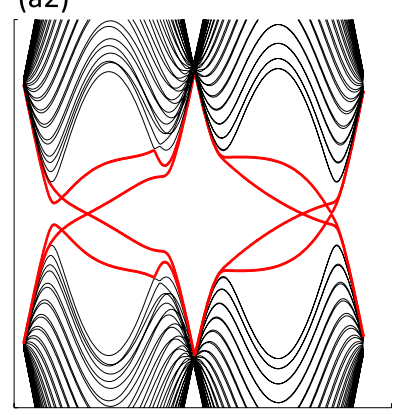

(b2)

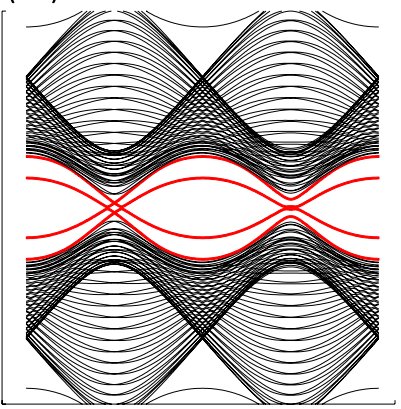

(a3)

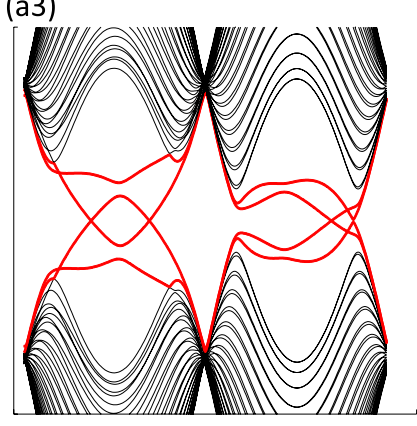

(b3)

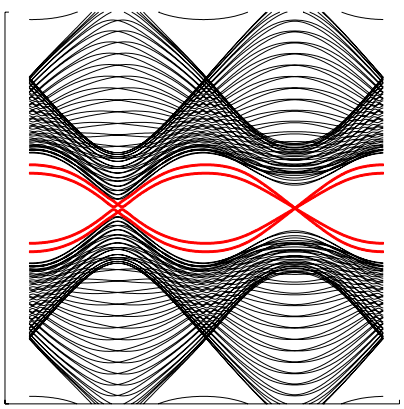

FIG. 7. (Color online) (a1)-(a3) [(b1)-(b3)] Band structures of Hamiltonian (1) for the zigzag [armchair] ribbon geometry with 40 [60] sites in the $y[x]$ direction with parameters $\left(\delta, t_{1}, h\right)=(0.1,0.03,0.11),(0.1,0.03,0.18)$, and $(0.1,0.1,0.07)$, respectively. These three cases show the edge states of the QAH effect with the Chern number of the whole system $C=-1,+1$, and +2 . Here, $k_{x}$ and $k_{y}$ are measured in units of $2 \pi / 3$. 
(a)

(b)

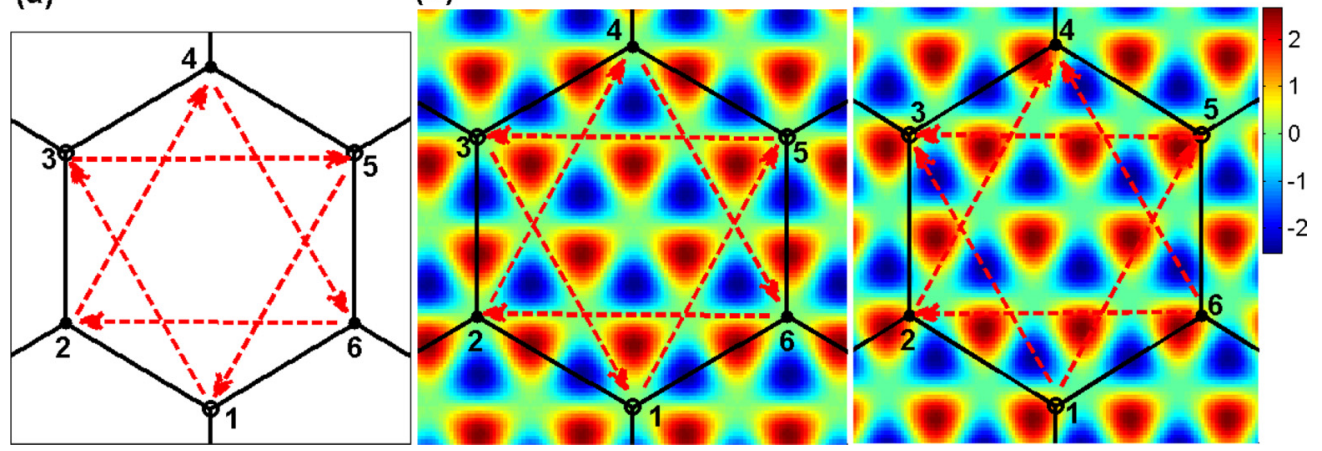

FIG. 8. (Color online) Illustration of the contours of the spin-dependent effective magnetic field. Arrows between the next-nearest-neighbor sites denote the directions of positive phase hopping for down-spin electrons. (a) The Haldane model; (b) the parameters $\gamma_{s=1,3}=\frac{\pi}{2}, \gamma_{2}=-\frac{\pi}{2}$, and $\phi=\pi / 2 ;$ (c) the parameters $\gamma_{s=1,2,3}=\frac{\pi}{2}$ and $\phi=2 \pi / 3$.

the gradient of the electric potential, i.e., the electric-field intensity. Similarly, in the ultracold-atom system, the Hall conductance corresponds to the response coefficient of the atom mass current about the gradient of the optical trapping potential. Thus, a nonzero Hall conductance gives the experimental signatures of the mass current and accumulations of atoms at the boundary of the optical lattice when the trapping potential is modulated.

In summary, we study quantum Hall effects in a nonAbelian honeycomb optical lattice which is a multi-Diracpoint system. The Hall conductance takes different values with the change in relative strengths of several perturbations. We find that gauge-field-dressed NNN hopping can induce the QSH effect, and a Zeeman field can induce the so-called quantum anomalous valley Hall effect, which includes two copies of quantum Hall states with opposite Chern numbers and counter-propagating edge states. The coexistence of both perturbations gives the nonzero Hall conductance, which is characterized by different Chern numbers. Our study shows the power of extended internal valley degrees of freedom in driving abundant quantum Hall effects in a non-Abelian honeycomb optical lattice.

\section{ACKNOWLEDGMENTS}

G. Liu was supported by NSF of China (No. 11247011), This work was supported by the NSFC under Grants No. 11434015, No. 61227902, No. 61378017, No. 11374354, No. 11174360, No. 11575051, and No. 51201057, NKBRSFC under Grants No. 2011CB921502 and No. 2012CB821305, SKLQOQOD under Grant No. KF201403, SPRPCAS under Grant No. XDB01020300, and Hebei NSF under Grants No. A2012202022 and No. A2015208024. N. Hao is supported by the Research Grant Council of Hong Kong under Grant No. HKU703713P.

\section{APPENDIX: SIMULATING THE GAUGE-FIELD-DRESSED NEXT-NEAREST-NEIGHBOR HOPPING}

In this section, we focus on how to simulate the gaugefield-dressed NNN hopping, i.e., the first term in Eq. (3), in our model in optical lattices. The first term in Eq. (3) actually corresponds to a specific spin-orbit coupling if we take $\gamma_{s=1,2,3}= \pm \frac{\pi}{2}$. It is well known that in the Haldane model [3], a periodic vector potential applied to a two-dimensional honeycomb lattice with zero net flux through each unit cell. The spacial magnetic flux density does not change the nearestneighbor hopping amplitude, but it causes the NNN hopping to have a chirality and breaks the time-reversal symmetry [see Fig. 8(a)]. Subsequently, Kane and Mele extended this mechanism to the time-reversal-invariant system, with up-spin and down-spin electrons having opposite chirality when they are hopping between NNN sites [4]. This kind of spindependent effective magnetic field is the intrinsic spin-orbit coupling in the Kane and Mele model. In Fig. 8, arrows between NNN sites denote the directions of positive phase hopping for down-spin electrons. The parameters $\gamma_{s=1,3}=\frac{\pi}{2}$ and $\gamma_{2}=-\frac{\pi}{2}$ correspond to the spin-dependent effective magnetic field in Fig. 8(b) and $\gamma_{s=1,2,3}=\frac{\pi}{2}$ correspond to Fig. 8(c). It is surprising that these two kinds of effective magnetic fields can give similar results for the system, and the latter corresponds to the results in the text. For comparison, we also show the Haldane model or Kane-Mele model in Fig. 8(a).

The spacial periodic magnetic field in the Haldane model can be simulated by use of the laser-induced-gauge-field method in an optical lattice [27]. With a similar method, we can design two other kinds of effective magnetic field. To this end, we consider a cold-atomic system with each atom having a $\Lambda$-type level configuration [11]. The ground states $|1\rangle$ and $|2\rangle$ are coupled to the excited state $|3\rangle$ through a spatially varying standing-wave laser field, with Rabi frequencies $\Omega_{p}=\Omega \sin \theta e^{-i S_{1}}$ and $\Omega_{c}=\Omega \cos \theta e^{-i S_{2}}$, respectively. With the rotating-wave approximation, the laser-atom coupling Hamiltonian is given by

$$
\hat{H}_{\text {int }}=-\frac{\hbar}{2}\left(\begin{array}{ccc}
0 & 0 & \Omega_{p} \\
0 & 0 & \Omega_{c} \\
\Omega_{p}^{*} & \Omega_{c}^{*} & -2 \Delta
\end{array}\right),
$$

with eigenstates (dressing states)

$$
\begin{gathered}
\left|\chi_{1}\right\rangle=e^{-i S_{1}} \cos \theta|1\rangle-e^{-i S_{2}} \sin \theta|2\rangle, \\
\left|\chi_{2}\right\rangle=\cos \varphi \sin \theta e^{-i S_{1}}|1\rangle+\cos \varphi \cos \theta e^{-i S_{2}}|2\rangle-\sin \varphi|3\rangle, \\
\left|\chi_{3}\right\rangle=\sin \varphi \sin \theta e^{-i S_{1}}|1\rangle+\sin \varphi \cos \theta e^{-i S_{2}}|2\rangle+\cos \varphi|3\rangle,
\end{gathered}
$$


and eigenvalues $\lambda_{n=1,2,3}=0, \frac{\hbar}{2}\left(\Delta \mp \sqrt{\Delta^{2}+\Omega^{2}}\right)$. Here, the single-photon detuning $\Delta=\omega_{3}-\omega_{1}-\omega_{p}$, with $\omega_{3}, \omega_{1}$, and $\omega_{p}$ the intrinsic frequency of atom state $|3\rangle$, atom state $|1\rangle$, and laser $\Omega_{p}$, respectively. In the new basis space $|\chi\rangle=$ $\left\{\left|\chi_{1}\right\rangle,\left|\chi_{2}\right\rangle,\left|\chi_{3}\right\rangle\right\}$, the primary atom Hamiltonian $\hat{H}=\frac{\mathbf{p}^{2}}{2 M}+$ $\hat{H}_{\text {int }}(\mathbf{r})+\hat{V}(\mathbf{r})$ can be rewritten as $H=\frac{1}{2 M}(-i \hbar \nabla-\mathbf{A})^{2}+V$, with $M$ the atom mass and $\mathbf{A}$ and $V$ the matrix with matrix elements $\mathbf{A}_{n, m}=i \hbar\left\langle\chi_{n}(\mathbf{r}) \mid \nabla \chi_{m}(\mathbf{r})\right\rangle$ and $V_{n, m}=\lambda_{n}(\mathbf{r}) \delta_{n, m}+$ $\left\langle\chi_{n}(\mathbf{r})|\hat{V}(\mathbf{r})| \chi_{m}(\mathbf{r})\right\rangle$, respectively. One can see that in the new basis the atom can be considered as moving in gauge potential $\mathbf{A}$, which corresponds to an effective magnetic field $\mathbf{B}_{\text {eff }}=(\nabla \times \mathbf{A})-\frac{i}{\hbar}(\mathbf{A} \times \mathbf{A})[27,28]$.

We focus on the subspace spanned by the two lower eigenstates $\left\{\left|\chi_{1}\right\rangle,\left|\chi_{2}\right\rangle\right\}$, which is redefined by $\left|\chi_{\uparrow}\right\rangle \equiv\left|\chi_{1}\right\rangle$ and $\left|\chi_{\downarrow}\right\rangle \equiv\left|\chi_{2}\right\rangle$ in the spin language. This gives an effective spin- $1 / 2$ system. For the large-detuning $(\Delta \gg \Omega)$ case, both state $\left|\chi_{\uparrow}\right\rangle$ and state $\left|\chi_{\downarrow}\right\rangle$ are stable under atomic spontaneous emission from the initial excite state $|3\rangle$. Furthermore, we assume the adiabatic condition, which requires that the off-diagonal elements of matrices $\mathbf{A}$ are smaller than the eigenenergy differences $\left|\lambda_{m}-\lambda_{n}\right|(m, n=1,2,3)$ of states $\left|\chi_{m}\right\rangle$. Under this adiabatic condition and in the basis space $\left\{\left|\chi_{\uparrow}\right\rangle,\left|\chi_{\downarrow}\right\rangle\right\}$, the gauge potential $\mathbf{A}$ becomes diagonal and has the form [29]

$$
\begin{aligned}
& \mathbf{A}=\left(\begin{array}{cc}
\mathbf{A}_{\uparrow} & 0 \\
0 & \mathbf{A}_{\downarrow}
\end{array}\right) \\
& \mathbf{A}_{\text {eff }}^{\alpha}=\alpha \hbar k_{1}\left[\left(\cos \left(2 k_{2} y-2 \phi\right)-\cos \left(k_{2} y+2 \phi\right) \cos \left(\sqrt{3} k_{2} x\right)\right) \mathbf{e}_{x}-\sqrt{3} \hbar k_{1} \sin \left(k_{2} y+2 \phi\right) \sin \left(\sqrt{3} k_{2} x\right) \mathbf{e}_{y}\right] \text {, } \\
& \mathbf{B}_{\mathrm{eff}}^{\alpha}=-\alpha 4 \pi \hbar k_{1}\left[2 \sin (2 \pi y+2 \phi) \cos \left(\frac{6 \pi}{\sqrt{3}} x\right)-\sin (4 \pi y-2 \phi)\right] \mathbf{e}_{z} .
\end{aligned}
$$

The total accumulated phases for nearest-neighbor hopping are 0 , and those for the NNN hopping along the arrowed directions in Fig. 8(b) are

$$
\begin{aligned}
\varphi_{13}^{\alpha} & =\varphi_{35}^{\alpha}=\varphi_{51}^{\alpha}=-\varphi_{24}^{\alpha}=-\varphi_{46}^{\alpha} \\
& =-\varphi_{62}^{\alpha}=\alpha \sqrt{3} k_{1} \cos (2 \phi) .
\end{aligned}
$$

The contours of the magnetic field with $\phi=\pi / 2$ for down-spin are also plotted in Fig. 8(b).

Comparing Fig. 8(b) with Fig. 8(c), we find that the phase factors have different signs along the sites $(1 \rightarrow 3)$ direction and the latter does not have $C_{3}$ rotational symmetry. So, along this direction, we select the Rabi frequencies $\Omega_{p}=\Omega \sin \left(-\frac{\sqrt{3}}{2} k_{2} x-\frac{1}{2} k_{2} y-\phi\right) e^{i\left(-\frac{1}{2} k_{1} x+\frac{\sqrt{3}}{2} k_{1} y\right)}$ and $\Omega_{c}=\Omega \cos \left(-\frac{\sqrt{3}}{2} k_{2} x-\frac{1}{2} k_{2} y-\phi\right) e^{-i\left(-\frac{1}{2} k_{1} x+\frac{\sqrt{3}}{2} k_{1} y\right)}$ and still set $k_{2} a=2 \pi$. The laser field along the $(1 \rightarrow 3)$ direction brings the nonzero phase factor $\varphi_{13}=-\varphi_{46}=$ $-\alpha \sqrt{3} k_{1} a \cos (2 \phi)$. Finally, the total effective vector potential and magnetic field for the Fig. 8(c) can be written as

$$
\begin{aligned}
& \text { A } \begin{aligned}
\mathbf{A}_{\mathrm{eff}}= & \hbar k_{1}\left[\cos \left(2 k_{2} y-2 \phi\right)-\sin \left(k_{2} y+2 \phi\right) \sin \left(\sqrt{3} k_{2} x\right)\right] \mathbf{e}_{x} \\
- & \sqrt{3} \hbar k_{1} \cos \left(k_{2} y+2 \phi\right) \cos \left(\sqrt{3} k_{2} x\right) \mathbf{e}_{y} \\
\mathbf{B}_{\mathrm{eff}}^{\alpha}= & \alpha 4 \hbar k_{1} \pi\left[2 \cos \left(\frac{2 \pi}{a} y+2 \phi\right) \sin \left(\frac{2 \sqrt{3} \pi}{a} x\right)\right. \\
& \left.+2 \sin \left(\frac{4 \pi}{a} y-2 \phi\right)\right] \mathbf{e}_{z} .
\end{aligned}
\end{aligned}
$$

The phase factors along the arrowed direction in Fig. 8(c) are $\varphi_{35}^{\alpha}=\varphi_{51}^{\alpha}=-\varphi_{13}^{\alpha}=\varphi_{46}^{\alpha}=-\varphi_{24}^{\alpha}=-\varphi_{62}^{\alpha}=\alpha \sqrt{3} k_{1} \cos (2 \phi)$

The contours of the magnetic field with $\phi=2 \pi / 3$ for downspin are plotted in Fig. 8(c).
[1] P. Wallace, Phys. Rev. 71, 622 (1947).

[2] G. W. Semenoff, Phys. Rev. Lett. 53, 2449 (1984).

[3] F. D. M. Haldane, Phys. Rev. Lett. 61, 2015 (1988).
[4] C. L. Kane and E. J. Mele, Phys. Rev. Lett. 95, 146802 (2005); 95, 226801 (2005).

[5] M. Z. Hasan and C. L. Kane, Rev. Mod. Phys. 82, 3045 (2010). 
[6] X. L. Qi and S. C. Zhang, Rev. Mod. Phys. 83, 1057 (2011).

[7] J. R. Anglin and W. Ketterle, Nature (London) 416, 211 (2002).

[8] D. Jaksch, C. Bruder, J. I. Cirac, C. W. Gardiner, and P. Zoller, Phys. Rev. Lett. 81, 3108 (1998).

[9] L. M. Duan, E. Demler, and M. D. Lukin, Phys. Rev. Lett. 91, 090402 (2003).

[10] T. Uehlinger, G. Jotzu, M. Messer, D. Greif, W. Hofstetter, U. Bissbort, and T. Esslinger, Phys. Rev. Lett. 111, 185307 (2013).

[11] G. Liu, S.-L. Zhu, S. Jiang, F. Sun, and W. M. Liu, Phys. Rev. A 82, 053605 (2010).

[12] G. Liu, N. Hao, S.-L. Zhu, and W. M. Liu, Phys. Rev. A 86, 013639 (2012).

[13] N. Hao, G. Liu, N. Wu, J. Hu, and Y. Wang, Phys. Rev. A 87, 053609 (2013).

[14] M. S. Scheurer, S. Rachel, and P. P. Orth, Sci. Rep. 5, 8386 (2015).

[15] R. Jördens, N. Strohmaier, K. Günter, H. Moritz, and T. Esslinger, Nature 455, 204 (2008).

[16] U. Schneider, L. Hackermülller, S. Will, Th. Best, I. Bloch, T. A. Costi, R. W. Helmes, D. Rasch, and A. Rosch, Science 322, 1520 (2008).

[17] K. Osterloh, M. Baig, L. Santos, P. Zoller, and M. Lewenstein, Phys. Rev. Lett. 95, 010403 (2005).
[18] G. Jotzu, M. Messer, R. Desbuquois, M. Lebrat, T. Uehlinger, D. Greif, and T. Esslinger, Nature 515, 237 (2014).

[19] P. Wang, Z.-Q. Yu, Z. Fu, J. Miao, L. Huang, S. Chai, H. Zhai, and J. Zhang, Phys. Rev. Lett. 109, 095301 (2012).

[20] L. W. Cheuk, A. T. Sommer, Z. Hadzibabic, T. Yefsah, W. S. Bakr, and M. W. Zwierlein, Phys. Rev. Lett. 109, 095302 (2012).

[21] A. Bermudez, N. Goldman, A. Kubasiak, M. Lewenstein, and M. A. Martin-Delgado, New J. Phys. 12, 033041 (2010).

[22] D. Sticlet and F. Piéchon, Phys. Rev. B 87, 115402 (2013); D. Sticlet, F. Piéchon, J.-N. Fuchs, P. Kalugin, and P. Simon, ibid. 85, 165456 (2012).

[23] M. Ezawa, New J. Phys. 16, 065015 (2014).

[24] C. Bena and L. Simon, Phys. Rev. B 83, 115404 (2011).

[25] X.-T. An, Y.-Y. Zhang, J.-J. Liu, and S.-S. Li, Appl. Phys. Lett. 102, 043113 (2013).

[26] C. C. Liu, H. Jiang, and Y. G. Yao, Phys. Rev. B 84, 195430 (2011).

[27] L. B. Shao, S.-L. Zhu, L. Sheng, D. Y. Xing, and Z. D. Wang, Phys. Rev. Lett. 101, 246810 (2008).

[28] J. Ruseckas, G. Juzeliūnas, P. Öhberg, and M. Fleischhauer, Phys. Rev. Lett. 95, 010404 (2005).

[29] S.-L. Zhu, H. Fu, C.-J. Wu, S.-C. Zhang, and L.-M. Duan, Phys. Rev. Lett. 97, 240401 (2006). 